\title{
Observation of the Top Quark
}

S. Abachi, ${ }^{12}$ B. Abbott,${ }^{33}$ M. Abolins, ${ }^{23}$ B.S. Acharya,${ }^{40}$ I. Adam,${ }^{10}$ D.L. Adams,${ }^{34}$ M. Adams, ${ }^{15}$ S. Ahn, ${ }^{12}$ H. Aihara, ${ }^{20}$ J. Alitti,${ }^{36}$ G. Álvarez, ${ }^{16}$ G.A. Alves ${ }^{8}$ E. Amidi, ${ }^{27}$ N. Amos,${ }^{22}$ E.W. Anderson, ${ }^{17}$ S.H. Aronson, ${ }^{3}$ R. Astur,${ }^{38}$ R.E. Avery,${ }^{29}$ A. Baden,${ }^{21}$ V. Balamurali, ${ }^{30}$ J. Balderston, ${ }^{14}$ B. Baldin,${ }^{12}$ J. Bantly, ${ }^{4}$ J.F. Bartlett,${ }^{12}$ K. Bazizi,${ }^{7}$ J. Bendich, ${ }^{20}$ S.B. Beri, ${ }^{31}$ I. Bertram,${ }^{34}$ V.A. Bezzubov,${ }^{32}$ P.C. Bhat, ${ }^{12}$ V. Bhatnagar, ${ }^{31}$ M. Bhattacharjee, ${ }^{11}$ A. Bischoff, ${ }^{7}$ N. Biswas, ${ }^{30}$ G. Blazey, ${ }^{12}$ S. Blessing, ${ }^{13}$ A. Boehnlein, ${ }^{12}$ N.I. Bojko, ${ }^{32}$ F. Borcherding, ${ }^{12}$ J. Borders,${ }^{35}$ C. Boswell, ${ }^{7}$ A. Brandt, ${ }^{12}$ R. Brock,${ }^{23}$ A. Bross, ${ }^{12}$ D. Buchholz, ${ }^{29}$ V.S. Burtovoi,${ }^{32}$ J.M. Butler, ${ }^{12}$ D. Casey, ${ }^{35}$ H. Castilla-Valdez,${ }^{9}$ D. Chakraborty, ${ }^{38}$ S.-M. Chang, ${ }^{27}$ S.V. Chekulaev ${ }^{32}$ L.-P. Chen, ${ }^{20}$ W. Chen, ${ }^{38}$ L. Chevalier, ${ }^{36}$ S. Chopra, ${ }^{31}$ B.C. Choudhary,${ }^{7}$ J.H. Christenson, ${ }^{12}$ M. Chung, ${ }^{15}$ D. Claes,${ }^{38}$ A.R. Clark, ${ }^{20}$ W.G. Cobau,${ }^{21}$ J. Cochran,${ }^{7}$ W.E. Cooper, ${ }^{12}$ C. Cretsinger,${ }^{35}$

D. Cullen-Vidal, ${ }^{4}$ M. Cummings, ${ }^{14}$ D. Cutts,,${ }^{4}$ O.I. Dahl,${ }^{20}$ K. De,${ }^{41}$ M. Demarteau, ${ }^{12}$ R. Demina,${ }^{27}$ K. Denisenko, ${ }^{12}$ N. Denisenko, ${ }^{12}$ D. Denisov, ${ }^{12}$ S.P. Denisov ${ }^{32}$ W. Dharmaratna, ${ }^{13}$ H.T. Diehl, ${ }^{12}$ M. Diesburg, ${ }^{12}$ G. Diloreto,${ }^{23}$ R. Dixon, ${ }^{12}$ P. Draper, ${ }^{41}$ J. Drinkard, ${ }^{6}$ Y. Ducros,${ }^{36}$ S.R. Dugad ${ }^{40}$ S. Durston-Johnson, ${ }^{35}$ D. Edmunds,${ }^{23}$ A.O. Efimov ${ }^{32}$ J. Ellison, ${ }^{7}$ V.D. Elvira, ${ }^{12, \ddagger}$ R. Engelmann, ${ }^{38}$ S. Eno, ${ }^{21}$ G. Eppley ${ }^{34}$ P. Ermolov, ${ }^{24}$ O.V. Eroshin,${ }^{32}$ V.N. Evdokimov, ${ }^{32}$ S. Fahey, ${ }^{23}$ T. Fahland, ${ }^{4}$ M. Fatyga,${ }^{3}$ M.K. Fatyga, ${ }^{35}$ J. Featherly, ${ }^{3}$ S. Feher, ${ }^{38}$ D. Fein, ${ }^{2}$ T. Ferbel,${ }^{35}$ G. Finocchiaro, ${ }^{38}$ H.E. Fisk ${ }^{12}$ Yu. Fisyak, ${ }^{24}$ E. Flattum,${ }^{23}$ G.E. Forden, ${ }^{2}$ M. Fortner, ${ }^{28}$ K.C. Frame,${ }^{23}$ P. Franzini ${ }^{10}$ S. Fredriksen, ${ }^{39}$ S. Fuess,${ }^{12}$ A.N. Galjaev ${ }^{32}$ E. Gallas, ${ }^{41}$ C.S. Gao, ${ }^{12, *}$

S. Gao, ${ }^{12, *}$ T.L. Geld, ${ }^{23}$ R.J. Genik II, ${ }^{23}$ K. Genser, ${ }^{12}$ C.E. Gerber, ${ }^{12, \S}$ B. Gibbard, ${ }^{3}$ M. Glaubman,${ }^{27}$ V. Glebov ${ }^{35}$ S. Glenn, ${ }^{5}$ J.F. Glicenstein, ${ }^{36}$ B. Gobbi, ${ }^{29}$ M. Goforth, ${ }^{13}$ A. Goldschmidt, ${ }^{20}$ B. Gomez, ${ }^{1}$ P.I. Goncharov ${ }^{32}$ H. Gordon, ${ }^{3}$ L.T. Goss, ${ }^{42}$ N. Graf,${ }^{3}$ P.D. Grannis, ${ }^{38}$ D.R. Green, ${ }^{12}$ J. Green,${ }^{28}$ H. Greenlee, ${ }^{12}$ G. Griffin,,${ }^{6}$ N. Grossman, ${ }^{12}$ P. Grudberg, ${ }^{20}$ S. Grünendahl, ${ }^{35}$ J.A. Guida, ${ }^{38}$ J.M. Guida, ${ }^{3}$ W. Guryn, ${ }^{3}$ S.N. Gurzhiev ${ }^{32}$ Y.E. Gutnikov, ${ }^{32}$ N.J. Hadley, ${ }^{21}$ H. Haggerty,${ }^{12}$ S. Hagopian,,${ }^{13}$ V. Hagopian, ${ }^{13}$ K.S. Hahn,${ }^{35}$ R.E. Hall,${ }^{6}$ S. Hansen, ${ }^{12}$ R. Hatcher, ${ }^{23}$ J.M. Hauptman, ${ }^{17}$ D. Hedin, ${ }^{28}$ A.P. Heinson, ${ }^{7}$ U. Heintz, ${ }^{12}$ R. Hernandez-Montoya, ${ }^{9}$ T. Heuring, ${ }^{13}$ R. Hirosky,,${ }^{13}$ J.D. Hobbs,${ }^{12}$ B. Hoeneisen,,${ }^{1}$ J.S. Hoftun, ${ }^{4}$ F. Hsieh,${ }^{22}$ Ting Hu,${ }^{38}$ Tong Hu, ${ }^{16}$ T. Huehn, ${ }^{7}$

S. Igarashi, ${ }^{12}$ A.S. Ito, ${ }^{12}$ E. James, ${ }^{2}$ J. Jaques, ${ }^{30}$ S.A. Jerger, ${ }^{23}$ J.Z.-Y. Jiang, ${ }^{38}$

T. Joffe-Minor, ${ }^{29}$ H. Johari, ${ }^{27}$ K. Johns, ${ }^{2}$ M. Johnson, ${ }^{12}$ H. Johnstad, ${ }^{39}$ A. Jonckheere, ${ }^{12}$ H. Jöstlein, ${ }^{12}$ S.Y. Jun,${ }^{29}$ C.K. Jung, ${ }^{38}$ S. Kahn, ${ }^{3}$ J.S. Kang, ${ }^{18}$ R. Kehoe, ${ }^{30}$ M. Kelly, ${ }^{30}$ A. Kernan,${ }^{7}$ L. Kerth,${ }^{20}$ C.L. Kim, ${ }^{18}$ S.K. Kim, ${ }^{37}$ A. Klatchko, ${ }^{13}$ B. Klima, ${ }^{12}$

B.I. Klochkov, ${ }^{32}$ C. Klopfenstein, ${ }^{38}$ V.I. Klyukhin, ${ }^{32}$ V.I. Kochetkov, ${ }^{32}$ J.M. Kohli, ${ }^{31}$ D. Koltick, ${ }^{33}$ A.V. Kostritskiy, ${ }^{32}$ J. Kotcher, ${ }^{3}$ J. Kourlas, ${ }^{26}$ A.V. Kozelov ${ }^{32}$

E.A. Kozlovski, ${ }^{32}$ M.R. Krishnaswamy,${ }^{40}$ S. Krzywdzinski, ${ }^{12}$ S. Kunori, ${ }^{21}$ S. Lami, ${ }^{38}$ G. Landsberg, ${ }^{38}$ R.E. Lanou, ${ }^{4}$ J-F. Lebrat, ${ }^{36}$ J. Lee-Franzini, ${ }^{38}$ A. Leflat ${ }^{24} \mathrm{H} . \mathrm{Li},{ }^{38} \mathrm{~J}$. Li, ${ }^{41}$ Y.K. Li ${ }^{29}$ Q.Z. Li-Demarteau, ${ }^{12}$ J.G.R. Lima,${ }^{8}$ D. Lincoln, ${ }^{22}$ S.L. Linn, ${ }^{13}$ J. Linnemann,${ }^{23}$ R. Lipton, ${ }^{12}$ Y.C. Liu,${ }^{29}$ F. Lobkowicz,${ }^{35}$ S.C. Loken, ${ }^{20}$ S. Lökös,${ }^{38}$ L. Lueking, ${ }^{12}$

A.L. Lyon,${ }^{21}$ A.K.A. Maciel,${ }^{8}$ R.J. Madaras,${ }^{20}$ R. Madden ${ }^{13}$ I.V. Mandrichenko, ${ }^{32}$ Ph. Mangeot, ${ }^{36}$ S. Mani,${ }^{5}$ B. Mansoulié, ${ }^{36}$ H.S. Mao, ${ }^{12, *}$ S. Margulies, ${ }^{15}$ R. Markeloff, ${ }^{28}$ L. Markosky, ${ }^{2}$ T. Marshall, ${ }^{16}$ M.I. Martin, ${ }^{12}$ M. Marx,${ }^{38}$ B. May, ${ }^{29}$ A.A. Mayorov,${ }^{32}$ 
R. McCarthy ${ }^{38}$ T. McKibben, ${ }^{15}$ J. McKinley, ${ }^{23}$ H.L. Melanson, ${ }^{12}$ J.R.T. de Mello Neto, ${ }^{8}$ K.W. Merritt ${ }^{12}$ H. Miettinen,${ }^{34}$ A. Milder,${ }^{2}$ C. Milner ${ }^{39}$ A. Mincer,${ }^{26}$ J.M. de Miranda,${ }^{8}$ C.S. Mishra, ${ }^{12}$ M. Mohammadi-Baarmand, ${ }^{38}$ N. Mokhov, ${ }^{12}$ N.K. Mondal ${ }^{40}$ H.E. Montgomery ${ }^{12}$ P. Mooney, ${ }^{1}$ M. Mudan,${ }^{26}$ C. Murphy,${ }^{16}$ C.T. Murphy,${ }^{12}$ F. Nang, ${ }^{4}$ M. Narain, ${ }^{12}$ V.S. Narasimham, ${ }^{40}$ A. Narayanan, ${ }^{2}$ H.A. Neal, ${ }^{22}$ J.P. Negret, ${ }^{1}$ E. Neis,${ }^{22}$ P. Nemethy, ${ }^{26}$ D. Nešić ${ }^{4}$ D. Norman ${ }^{42}$ L. Oesch,${ }^{22}$ V. Oguri ${ }^{8}$ E. Oltman, ${ }^{20}$ N. Oshima,${ }^{12}$ D. Owen, ${ }^{23}$ P. Padley ${ }^{34}$ M. Pang,${ }^{17}$ A. Para,${ }^{12}$ C.H. Park,${ }^{12}$ Y.M. Park,${ }^{19}$ R. Partridge,${ }^{4}$ N. Parua,${ }^{40}$ M. Paterno, ${ }^{35}$ J. Perkins, ${ }^{41}$ A. Peryshkin, ${ }^{12}$ M. Peters,${ }^{14}$ H. Piekarz,${ }^{13}$ Y. Pischalnikov ${ }^{33}$ A. Pluquet, ${ }^{36}$ V.M. Podstavkov ${ }^{32}$ B.G. Pope ${ }^{23}$ H.B. Prosper, ${ }^{13}$ S. Protopopescu, ${ }^{3}$ D. Pušeljić, ${ }^{20}$ J. Qian, ${ }^{22}$ P.Z. Quintas,${ }^{12}$ R. Raja, ${ }^{12}$ S. Rajagopalan,${ }^{38}$ O. Ramirez,${ }^{15}$ M.V.S. Rao, ${ }^{40}$ P.A. Rapidis, ${ }^{12}$ L. Rasmussen, ${ }^{38}$ A.L. Read ${ }^{12}$ S. Reucroft, ${ }^{27}$ M. Rijssenbeek, ${ }^{38}$ T. Rockwell, ${ }^{23}$ N.A. Roe,${ }^{20}$ J.M.R. Roldan, ${ }^{1}$ P. Rubinov, ${ }^{38}$ R. Ruchti, ${ }^{30}$ S. Rusin, ${ }^{24}$ J. Rutherfoord,${ }^{2}$ A. Santoro, ${ }^{8}$ L. Sawyer, ${ }^{41}$ R.D. Schamberger,${ }^{38}$ H. Schellman, ${ }^{29}$ D. Schmid, ${ }^{39}$ J. Sculli, ${ }^{26}$ E. Shabalina, ${ }^{24}$ C. Shaffer, ${ }^{13}$ H.C. Shankar, ${ }^{40}$ R.K. Shivpuri, ${ }^{11}$ M. Shupe, ${ }^{2}$ J.B. Singh,${ }^{31}$ V. Sirotenko, ${ }^{28}$ W. Smart,${ }^{12}$ A. Smith,${ }^{2}$ R.P. Smith, ${ }^{12}$ R. Snihur, ${ }^{29}$ G.R. Snow, ${ }^{25}$ S. Snyder, ${ }^{38}$ J. Solomon, ${ }^{15}$ P.M. Sood ${ }^{31}$ M. Sosebee, ${ }^{41}$ M. Souza, ${ }^{8}$ A.L. Spadafora, ${ }^{20}$ R.W. Stephens, ${ }^{41}$ M.L. Stevenson, ${ }^{20}$ D. Stewart, ${ }^{22}$ F. Stocker, ${ }^{39}$ D.A. Stoianova, ${ }^{32}$ D. Stoker, ${ }^{6}$ K. Streets, ${ }^{26}$ M. Strovink,${ }^{20}$ A. Taketani, ${ }^{12}$ P. Tamburello, ${ }^{21}$ J. Tarazi,${ }^{6}$ M. Tartaglia, ${ }^{12}$ T.L. Taylor, ${ }^{29}$ J. Teiger,${ }^{36}$ J. Thompson, ${ }^{21}$ T.G. Trippe,${ }^{20}$ P.M. Tuts, ${ }^{10}$ N. Varelas, ${ }^{23}$ E.W. Varnes,${ }^{20}$ P.R.G. Virador, ${ }^{20}$

D. Vititoe, ${ }^{2}$ A.A. Volkov, ${ }^{32}$ E. von Goeler,${ }^{27}$ A.P. Vorobiev,${ }^{32}$ H.D. Wahl, ${ }^{13}$ J. Wang, ${ }^{12, *}$

L.Z. Wang, ${ }^{12, *}$ J. Warchol,${ }^{30}$ M. Wayne,${ }^{30}$ H. Weerts,${ }^{23}$ W.A. Wenzel,${ }^{20}$ A. White,${ }^{41}$ J.T. White, ${ }^{42}$ J.A. Wightman,${ }^{17}$ J. Wilcox, ${ }^{27}$ S. Willis, ${ }^{28}$ S.J. Wimpenny, ${ }^{7}$ J.V.D. Wirjawan, ${ }^{42}$ Z. Wolf, ${ }^{39}$ J. Womersley, ${ }^{12}$ E. Won,${ }^{35}$ D.R. Wood, ${ }^{12}$ H. Xu,${ }^{4}$ R. Yamada,${ }^{12}$ P. Yamin, ${ }^{3}$ C. Yanagisawa,${ }^{38}$ J. Yang,${ }^{26}$ T. Yasuda,${ }^{27}$ C. Yoshikawa,${ }^{14}$ S. Youssef, ${ }^{13}$ J. Yu, ${ }^{35}$ Y. Yu, ${ }^{37}$ Y. Zhang, ${ }^{12, *}$ Y.H. Zhou, ${ }^{12, *}$ Q. Zhu, ${ }^{26}$ Y.S. Zhu, ${ }^{12, *}$ Z.H. Zhu, ${ }^{35}$ D. Zieminska, ${ }^{16}$ A. Zieminski, ${ }^{16}$ A. Zinchenko, ${ }^{17}$ and A. Zylberstejn ${ }^{36}$

(DØ Collaboration)

\author{
${ }^{1}$ Universidad de los Andes, Bogota, Colombia \\ ${ }^{2}$ University of Arizona, Tucson, Arizona 85721 \\ ${ }^{3}$ Brookhaven National Laboratory, Upton, New York 11973 \\ ${ }^{4}$ Brown University, Providence, Rhode Island 02912 \\ ${ }^{5}$ University of California, Davis, California 95616 \\ ${ }^{6}$ University of California, Irvine, California 92717 \\ ${ }^{7}$ University of California, Riverside, California 92521 \\ ${ }^{8}$ LAFEX, Centro Brasileiro de Pesquisas Físicas, Rio de Janeiro, Brazil \\ ${ }^{9}$ CINVESTAV, Mexico City, Mexico \\ ${ }^{10}$ Columbia University, New York, New York 10027 \\ ${ }^{11}$ Delhi University, Delhi, India 110007 \\ ${ }^{12}$ Fermi National Accelerator Laboratory, Batavia, Illinois 60510 \\ ${ }^{13}$ Florida State University, Tallahassee, Florida 32306 \\ ${ }^{14}$ University of Hawaii, Honolulu, Hawaii 96822 \\ ${ }^{15}$ University of Illinois, Chicago, Illinois 60680
}


${ }^{16}$ Indiana University, Bloomington, Indiana 47405

${ }^{17}$ Iowa State University, Ames, Iowa 50011

${ }^{18}$ Korea University, Seoul, Korea

${ }^{19}$ Kyungsung University, Pusan, Korea

${ }^{20}$ Lawrence Berkeley Laboratory, Berkeley, California 94720

${ }^{21}$ University of Maryland, College Park, Maryland 20742

${ }^{22}$ University of Michigan, Ann Arbor, Michigan 48109

${ }^{23}$ Michigan State University, East Lansing, Michigan 48824

${ }^{24}$ Moscow State University, Moscow, Russia

${ }^{25}$ University of Nebraska, Lincoln, Nebraska 68588

${ }^{26}$ New York University, New York, New York 10003

${ }^{27}$ Northeastern University, Boston, Massachusetts 02115

${ }^{28}$ Northern Illinois University, DeKalb, Illinois 60115

${ }^{29}$ Northwestern University, Evanston, Illinois 60208

${ }^{30}$ University of Notre Dame, Notre Dame, Indiana 46556

${ }^{31}$ University of Panjab, Chandigarh 16-00-14, India

${ }^{32}$ Institute for High Energy Physics, 142-284 Protvino, Russia

${ }^{33}$ Purdue University, West Lafayette, Indiana 47907

${ }^{34}$ Rice University, Houston, Texas 77251

${ }^{35}$ University of Rochester, Rochester, New York 14627

${ }^{36}$ CEA, DAPNIA/Service de Physique des Particules, CE-SACLAY, France

${ }^{37}$ Seoul National University, Seoul, Korea

${ }^{38}$ State University of New York, Stony Brook, New York 11794

${ }^{39}$ SSC Laboratory, Dallas, Texas 75237

${ }^{40}$ Tata Institute of Fundamental Research, Colaba, Bombay 400005, India

${ }^{41}$ University of Texas, Arlington, Texas 76019

${ }^{42}$ Texas A $\& M$ University, College Station, Texas 77843

(February 7, 2008)

\begin{abstract}
The D $\varnothing$ collaboration reports on a search for the Standard Model top quark in $p \bar{p}$ collisions at $\sqrt{s}=1.8 \mathrm{TeV}$ at the Fermilab Tevatron, with an integrated luminosity of approximately $50 \mathrm{pb}^{-1}$. We have searched for $t \bar{t}$ production in the dilepton and single-lepton decay channels, with and without tagging of $b$-quark jets. We observed 17 events with an expected background of $3.8 \pm 0.6$ events. The probability for an upward fluctuation of the background to produce the observed signal is $2 \times 10^{-6}$ (equivalent to 4.6 standard deviations). The kinematic properties of the excess events are consistent with top quark decay. We conclude that we have observed the top quark and measure its mass to be $199_{-21}^{+19}$ (stat.) \pm 22 (syst.) $\mathrm{GeV} / \mathrm{c}^{2}$ and its production cross section to be $6.4 \pm 2.2 \mathrm{pb}$.
\end{abstract}


In the Standard Model (SM), the top quark is the weak isospin partner of the $b$ quark. The D $\varnothing$ collaboration published a lower limit on the mass of the top quark of $131 \mathrm{GeV} / \mathrm{c}^{2}$, at a confidence level (CL) of 95\%, based on an integrated luminosity of $13.5 \mathrm{pb}^{-1}$ [臬] A subsequent publication [2] reported the top quark production cross section as a function of the assumed top quark mass. In that analysis, we found nine events with an expected background of $3.8 \pm 0.9$ events (statistical significance 1.9 standard deviations) corresponding to a production cross section of $8.2 \pm 5.1 \mathrm{pb}$ for an assumed top quark mass of $180 \mathrm{GeV} / \mathrm{c}^{2}$. The CDF collaboration published evidence for top quark production with a statistical significance of 2.8 standard deviations, a top quark of mass $174 \pm 10_{-12}^{+13} \mathrm{GeV} / \mathrm{c}^{2}$, and a production cross section of $13.9_{-4.8}^{+6.1} \mathrm{pb}$ [3]. Precision electroweak measurements predict a SM top quark mass of approximately $150-210 \mathrm{GeV} / \mathrm{c}^{2}$, depending on the mass of the Higgs boson [4]. In the present paper, we report new results from the $\mathrm{D} \varnothing$ experiment that firmly establish the existence of the top quark.

We assume that the top quark is pair-produced and decays according to the minimal $\mathrm{SM}$ (i.e. $t \bar{t} \rightarrow W^{+} W^{-} b \bar{b}$ ). We have searched for the top quark in channels where both $W$ bosons decayed leptonically $(e \mu+$ jets, $e e+$ jets, and $\mu \mu+$ jets $)$ and in channels where just one $W$ boson decayed leptonically ( $e+$ jets and $\mu+$ jets $)$. The single-lepton channels were subdivided into $b$-tagged and untagged channels according to whether or not a muon was observed consistent with $b \rightarrow \mu+X$. The muon-tagged channels are denoted $e+$ jets $/ \mu$ and $\mu+$ jets $/ \mu$.

Here we present an analysis based on data collected at the Fermilab Tevatron at $\sqrt{s}=$ $1.8 \mathrm{TeV}$ with an integrated luminosity of $44-56 \mathrm{pb}^{-1}$, depending on the channel. In the present analysis, the signal-to-background ratio for a high mass top quark was substantially improved relative to Ref. [2]. An optimization of the selection criteria was carried out using Monte Carlo top quark events for signal and our standard background estimates. The result of this procedure was a factor of 3.7 better background rejection while retaining $70 \%$ of the acceptance for $180 \mathrm{GeV} / \mathrm{c}^{2}$ top quarks. This corresponds to a signal-to-background ratio of 1:1 for a top quark mass of $200 \mathrm{GeV} / \mathrm{c}^{2}$, assuming the expected SM top cross section [5]. The improved rejection arises primarily by requiring events to have a larger total transverse energy.

The D $\varnothing$ detector and data collection systems are described in Ref. [6]. The triggers and reconstruction algorithms for jets, electrons, muons, and neutrinos were the same as those used in our previous top quark searches [1:2].

The signature for the dilepton channels was defined as two isolated leptons, at least two jets, and large missing transverse energy $E_{T}$. The signature for the single-lepton channels was defined as one isolated lepton, large $\not_{T}$, and a minimum of three jets (with muon tag) or four jets (without tag). The minimum transverse momentum $p_{T}$ of tagging muons was $4 \mathrm{GeV} / \mathrm{c}$. Requirements pertaining to the magnitude and direction of the $\not_{T}$, the aplanarity of the jets $\mathcal{A}$, and the allowed ranges of pseudorapidity $\eta$ were similar to Ref. [2]. Muons were restricted to $|\eta|<1$ for the last $70 \%$ of the data because of forward muon chamber aging. Events in the $\mu \mu+$ jets and $\mu+$ jets $/ \mu$ channels were required to be inconsistent with the $Z+$ jets hypothesis, based on a global kinematic fit. The principal difference between the present analysis and the analysis of Ref. [2] was the imposition of a minimum requirement in all channels on a quantity $H_{T}$, which we defined as the scalar sum of the transverse energies $E_{T}$ of the jets (for the single-lepton and $\mu \mu+$ jets channels) or the 
scalar sum of the $E_{T}$ 's of the leading electron and the jets (for the $e \mu+$ jets and $e e+$ jets channels). The kinematic requirements for our standard event selection for all seven channels

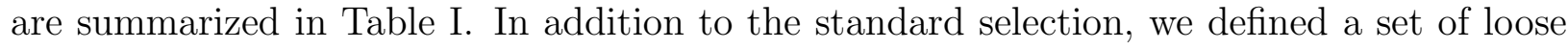
event selection requirements, which differed from the standard set by the removal of the $H_{T}$ requirement and by the relaxation of the aplanarity requirement for $e+$ jets and $\mu+$ jets from $\mathcal{A}>0.05$ to $\mathcal{A}>0.03$.

For the dilepton channels, the main backgrounds were from $Z$ and continuum Drell-Yan

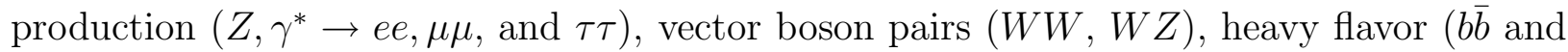
$c \bar{c})$ production, and backgrounds with jets misidentified as leptons. For the single-lepton channels, the main backgrounds were from $W+$ jets, $Z+$ jets, and multijet production with a jet misidentified as a lepton. The method for estimating these backgrounds was the same as in our previously published analyses [1,2].

$H_{T}$ is a powerful discriminator between background and high-mass top quark production. Figure 1 shows a comparison of the shapes of the $H_{T}$ distributions expected from background and $200 \mathrm{GeV} / \mathrm{c}^{2}$ top quarks in the channels (a) $e \mu+$ jets and (b) untagged single-lepton + jets. We have tested our understanding of background $H_{T}$ distributions by comparing data and calculated background in background-dominated channels such as electron + two jets and electron + three jets (Fig. 2). The observed $H_{T}$ distribution agrees with the background calculation, which includes contributions from both $W+$ jets as calculated by the VECBOS Monte Carlo [0] and multijet events.

The acceptance for $t \bar{t}$ events was calculated using the ISAJET event generator [8] and a detector simulation based on the GEANT program [9]. As a check, the acceptance was also calculated using the HERWIG event generator [10]. The difference between ISAJET and HERWIG was included in the systematic error.

From all seven channels, we observed 17 events with an expected background of $3.8 \pm 0.6$ events (see Table [I]). Our measured cross section as a function of the top quark mass hypothesis is shown in Fig. 3. Assuming a top quark mass of $200 \mathrm{GeV} / \mathrm{c}^{2}$, the production cross section is $6.3 \pm 2.2 \mathrm{pb}$. The error in the cross section includes an overall $12 \%$ uncertainty in the luminosity. The probability of an upward fluctuation of the background to 17 or more events is $2 \times 10^{-6}$, which corresponds to 4.6 standard deviations for a Gaussian probability distribution. We have calculated the probability for our observed distribution of excess events among the seven channels and find that our results are consistent with top quark branching fractions at the $53 \%$ CL. Thus, we observe a statistically significant excess of events and the distribution of events among the seven channels is consistent with top quark production.

Additional confirmation that our observed excess contains a high-mass object comes from the invariant masses of jet combinations in single-lepton + jets events. For this analysis, we selected single-lepton + four-jet events using the loose event selection requirements (27 events). An invariant mass analysis was performed, based on the hypothesis $t \bar{t} \rightarrow W^{+} W^{-} b \bar{b} \rightarrow \ell \nu q \bar{q} b \bar{b}$. One jet was assigned to the semileptonically decaying top quark and three jets were assigned to the hadronically decaying top quark. The jet assignment algorithm attempted to assign one of the two highest $E_{T}$ jets to the semileptonically decaying top quark and to minimize the difference between the masses of the two top quarks. The invariant mass of the three jets assigned to the hadronically decaying top quark is denoted by $m_{3 \mathrm{j}}$. The invariant mass of the pair of hadronically decaying top quark jets with the 
smallest invariant mass is denoted by $m_{2 \mathrm{j}}$. Figure 1 shows the distribution of $m_{3 \mathrm{j}} v s . m_{2 \mathrm{j}}$ for (a) background ( $W+$ jets and multijet) (b) $200 \mathrm{GeV} / \mathrm{c}^{2}$ top Monte Carlo, and (c) data. The data are peaked at higher invariant mass, in both dimensions, than the background. Based only on the shapes of the distributions, the hypothesis that the data are a combination of top quark and background events $(60 \% \mathrm{CL})$ is favored over the pure background hypothesis (3\% CL).

To measure the top quark mass, single-lepton + four-jet events were subjected to 2constraint kinematic fits to the hypothesis $t \bar{t} \rightarrow W^{+} W^{-} b \bar{b} \rightarrow \ell \nu q \bar{q} b \bar{b}$. Kinematic fits were performed on all permutations of the jet assignments of the four highest $E_{T}$ jets, with the provision that muon-tagged jets were always assigned to a $b$-quark in the fit. A maximum of three permutations with $\chi^{2}<7$ (two degrees of freedom) were retained, and a single $\chi^{2}$-probability-weighted average mass ("fitted mass") was calculated for each event. Monte Carlo studies using the ISAJET and HERWIG event generators showed that the fitted mass was strongly correlated with the top quark mass. Gluon radiation, jet assignment combinatorics, and the event selection procedure introduced a shift in the fitted mass (approximately $-20 \mathrm{GeV} / \mathrm{c}^{2}$ for $200 \mathrm{GeV} / \mathrm{c}^{2}$ top quarks), which was taken into account in the final mass determination.

Eleven of the 14 single-lepton + jets candidate events selected using the standard cuts were fitted successfully. Figure 5(a) shows the fitted mass distribution. An unbinned likelihood fit, incorporating top quark and background contributions, with the top quark mass allowed to vary, was performed on the fitted mass distribution. The top quark contribution was modeled using ISAJET. The background contributions were constrained to be consistent with our background estimates. The likelihood fit yielded a top quark mass of $199_{-25}^{+31}$ (stat.) $\mathrm{GeV} / \mathrm{c}^{2}$ and described the data well.

To increase the statistics available for the mass fit, and to remove any bias from the standard $H_{T}$ requirement, we repeated the mass analysis on events selected using the loose requirements. Of 27 single-lepton + four-jet events, 24 were fitted successfully. The removal of the $H_{T}$ requirement introduced a substantial background contribution at lower mass in addition to the top signal, as shown in Fig. 5(b). A likelihood fit to the mass distribution resulted in a top quark mass of $199_{-21}^{+19}$ (stat.) $\mathrm{GeV} / \mathrm{c}^{2}$, consistent with the result obtained from the standard event selection. The result of the likelihood fit did not depend significantly on whether the normalization of the background was constrained. Using HERWIG to model the top quark contribution resulted in a mass $4 \mathrm{GeV} / \mathrm{c}^{2}$ below that found using ISAJET. This effect was included in the systematic error. The total systematic error in the top quark mass is $22 \mathrm{GeV} / \mathrm{c}^{2}$, which is dominated by the uncertainty in the jet energy scale.

In conclusion, we report the observation of the top quark. We measure the top quark mass to be $199_{-21}^{+19}$ (stat.) \pm 22 (syst.) $\mathrm{GeV} / \mathrm{c}^{2}$ and measure a production cross section of $6.4 \pm 2.2 \mathrm{pb}$ at our central mass.

We thank the Fermilab Accelerator, Computing, and Research Divisions, and the support staffs at the collaborating institutions for their contributions to the success of this work. We also acknowledge the support of the U.S. Department of Energy, the U.S. National Science Foundation, the Commissariat à L'Energie Atomique in France, the Ministry for Atomic Energy and the Ministry of Science and Technology Policy in Russia, CNPq in Brazil, the Departments of Atomic Energy and Science and Education in India, Colciencias 
in Colombia, CONACyT in Mexico, the Ministry of Education, Research Foundation and KOSEF in Korea, and the A. P. Sloan Foundation. 


\section{REFERENCES}

* Visitor from IHEP, Beijing, China.

‡ Visitor from CONICET, Argentina.

$\S \quad$ Visitor from Universidad de Buenos Aires, Argentina.

ฯ Visitor from Univ. San Francisco de Quito, Ecuador.

[1] DØ Collaboration, S. Abachi et al., Phys. Rev. Lett. 72, 2138 (1994).

[2] DØ Collaboration, S. Abachi et al., FERMILAB-PUB-94/354-E, 1994 (to be published in Phys. Rev. Lett.); FERMILAB-PUB-95/020-E, 1995 (in preparation).

[3] CDF Collaboration, F. Abe et al., Phys. Rev. D 50, 2966 (1994); Phys. Rev. Lett. 73, 225 (1994).

[4] D. Schaile, CERN-PPE/94-162, presented at 27th International Conference on High Energy Physics, Glasgow, July 1994 (unpublished).

[5] E. Laenen, J. Smith, and W. van Neerven, Phys. Lett. 321B, 254 (1994).

[6] DØ Collaboration, S. Abachi et al., Nucl. Instrum. Methods A338, 185 (1994).

[7] W. Giele, E. Glover, and D. Kosower, Nucl. Phys. B403, 633 (1993).

[8] F. Paige and S. Protopopescu, BNL Report no. BNL38034, 1986 (unpublished), release v 6.49 .

[9] F. Carminati et al., "GEANT Users Guide," CERN Program Library, 1991 (unpublished).

[10] G. Marchesini et al., Comput. Phys. Commun. 67, 465 (1992). 


\section{FIGURES}

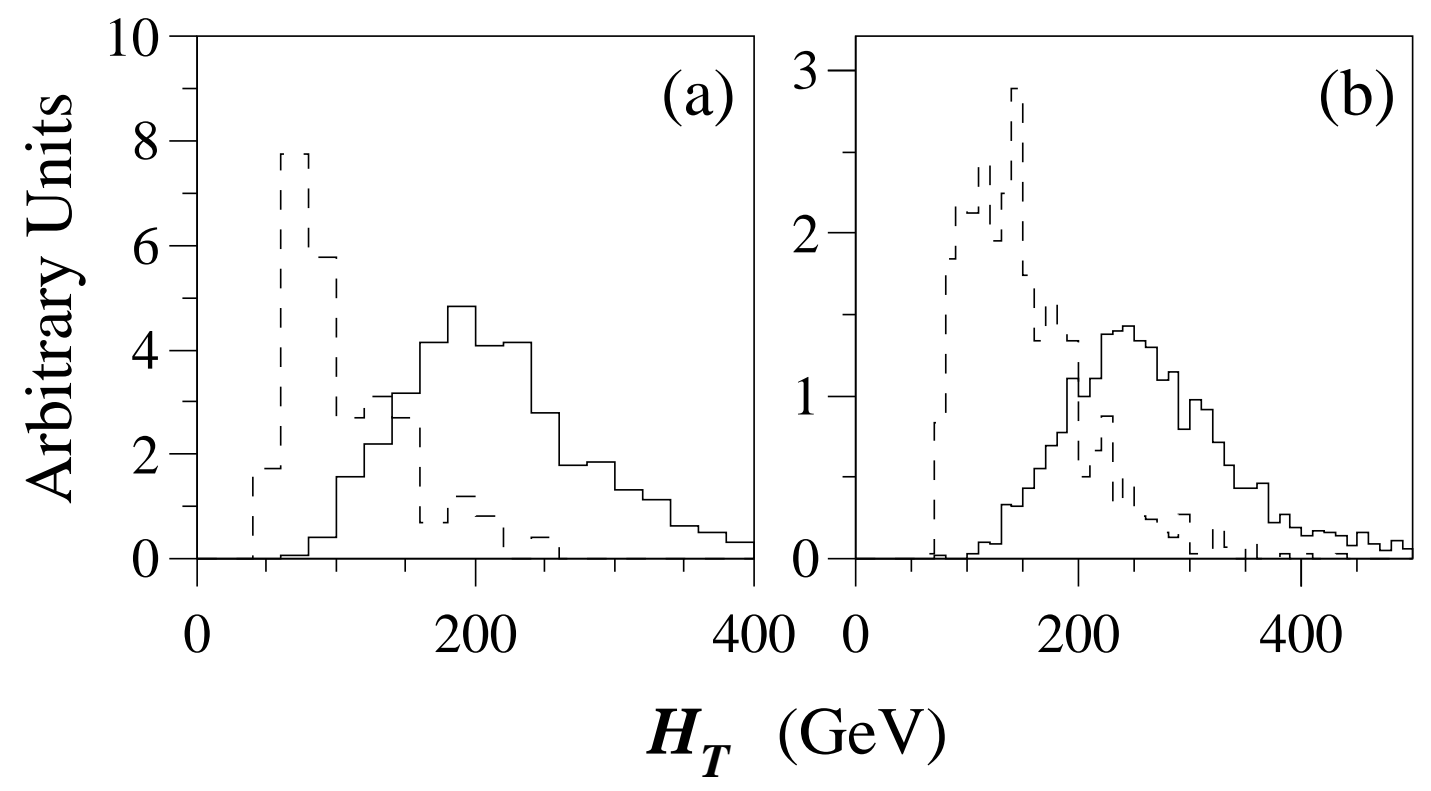

FIG. 1. Shape of $H_{T}$ distributions expected for the principal backgrounds (dashed line) and $200 \mathrm{GeV} / \mathrm{c}^{2}$ top quarks (solid line) for (a) $e \mu+$ jets and (b) untagged single-lepton + jets.

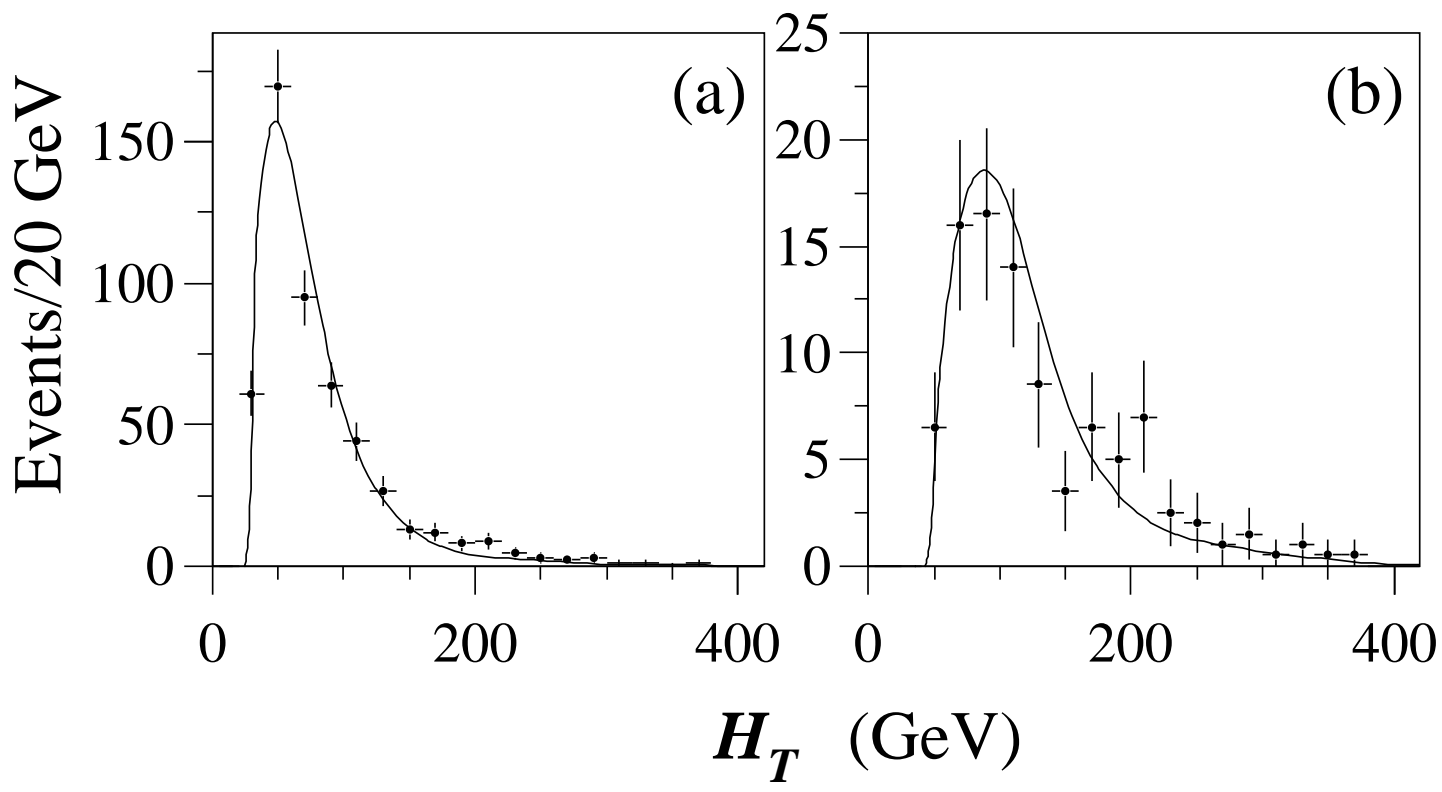

FIG. 2. Observed $H_{T}$ distributions (points) compared to the distributions expected from background (line) for $\not_{T}>25 \mathrm{GeV} / \mathrm{c}$ and (a) $e+\geq 2$ jets and (b) $e+\geq 3$ jets. 


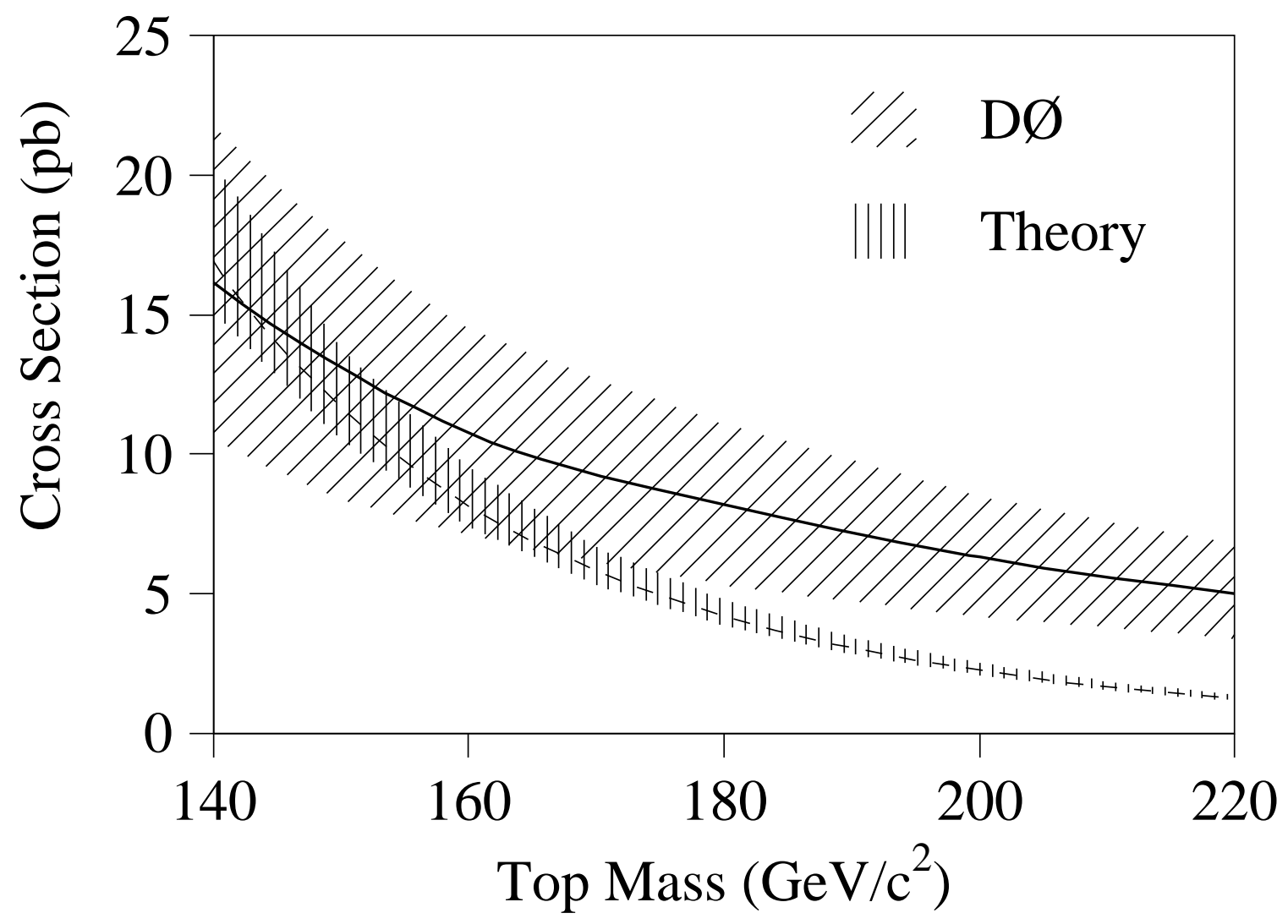

FIG. 3. DØ measured $t \bar{t}$ production cross section (solid line with one standard deviation error band) as a function of assumed top quark mass. Also shown is the theoretical cross section curve (dashed line) [5]. 
(a)

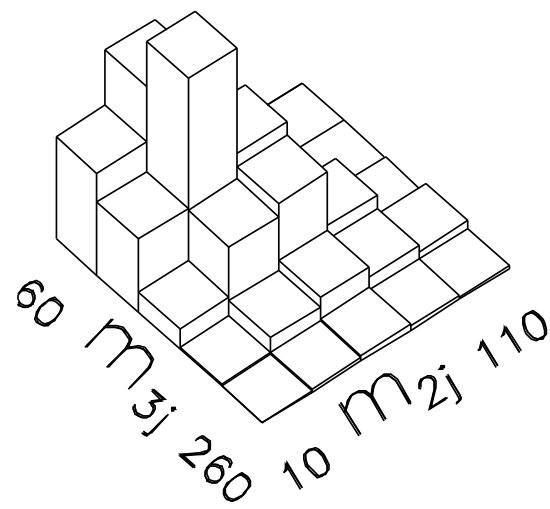

(b)

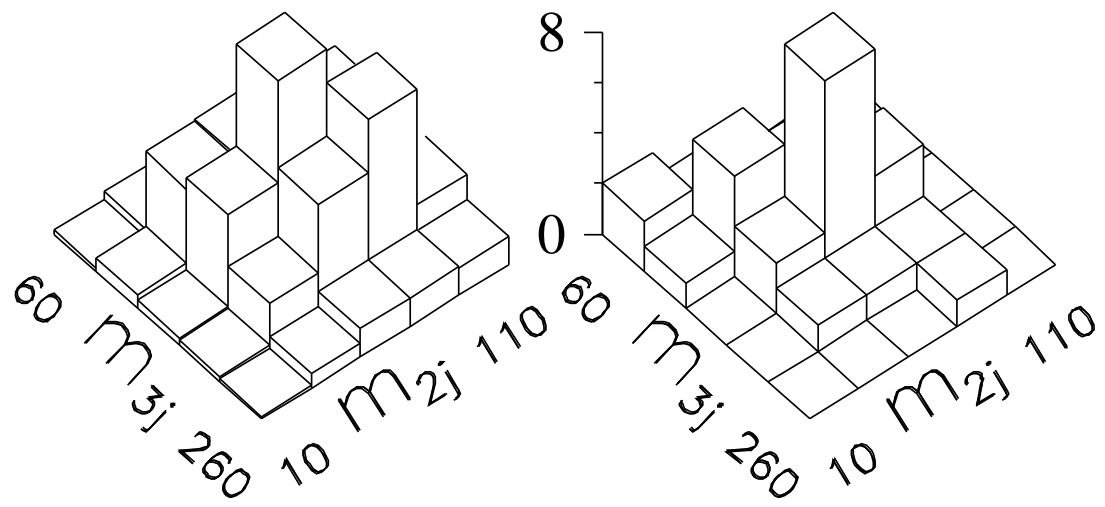

FIG. 4. Single-lepton + jets two-jet vs. three-jet invariant mass distribution for (a) background, (b) $200 \mathrm{GeV} / \mathrm{c}^{2}$ top Monte Carlo (ISAJET), and (c) data.

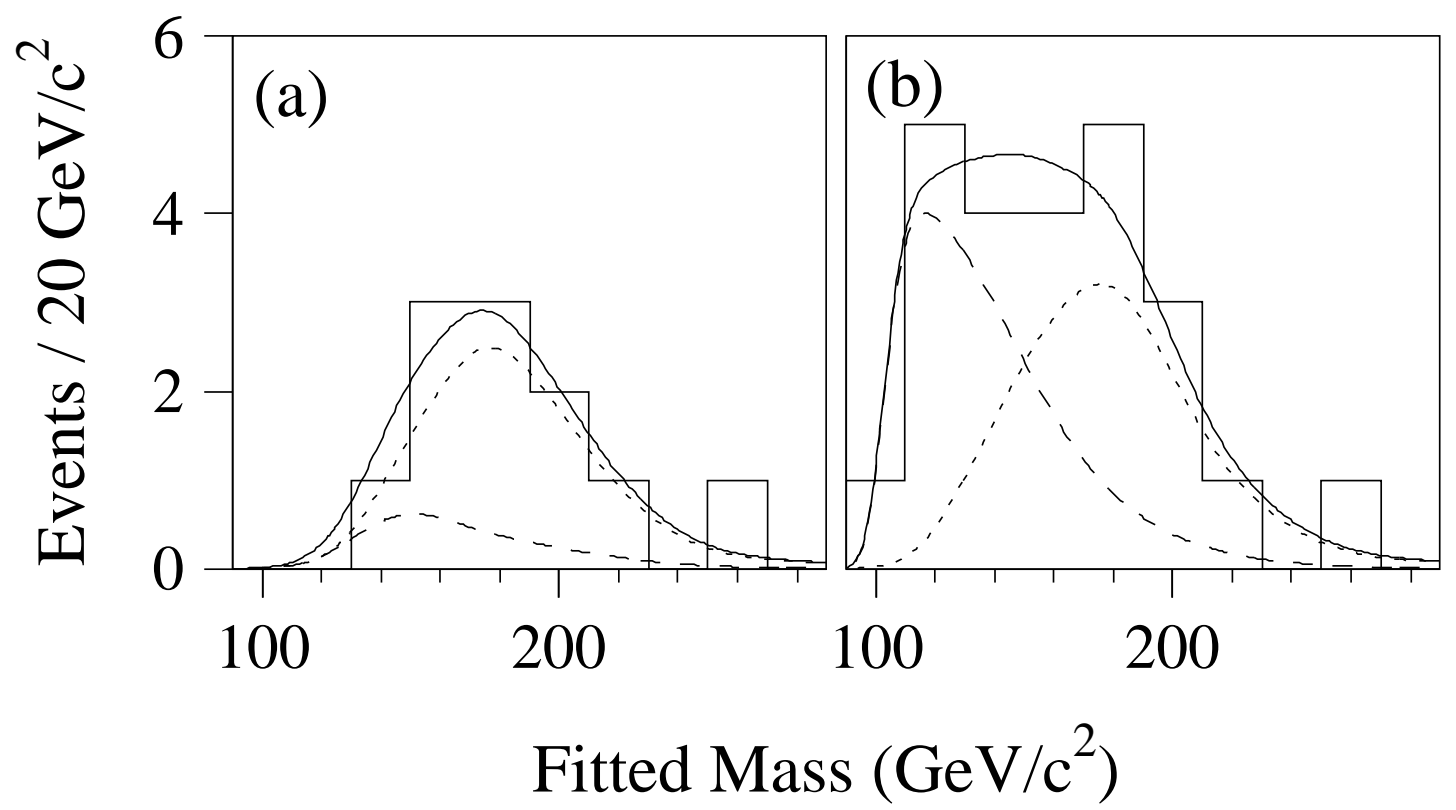

FIG. 5. Fitted mass distribution for candidate events (histogram) with the expected mass distribution for $199 \mathrm{GeV} / \mathrm{c}^{2}$ top quark events (dotted curve), background (dashed curve), and the sum of top and background (solid curve) for (a) standard and (b) loose event selection. 


\section{TABLES}

TABLE I. Minimum kinematic requirements for the standard event selection (energy in GeV).

\begin{tabular}{c|c|c|c|r|r|r|r}
\hline \hline & \multicolumn{2}{|c|}{ Leptons } & \multicolumn{2}{|c|}{ Jets } & & \multirow{2}{*}{ Channel } \\
\cline { 2 - 5 } & $E_{T}(e)$ & $p_{T}(\mu)$ & $N_{\text {jet }}$ & $E_{T}$ & $B_{T}$ & $H_{T}$ & $\mathcal{A}$ \\
\hline \hline$e \mu+$ jets & 15 & 12 & 2 & 15 & 20 & 120 & - \\
$e e+$ jets & 20 & & 2 & 15 & 25 & 120 & - \\
$\mu \mu+$ jets & & 15 & 2 & 15 & - & 100 & - \\
\hline$e+$ jets & 20 & & 4 & 15 & 25 & 200 & 0.05 \\
$\mu+$ jets & & 15 & 4 & 15 & 20 & 200 & 0.05 \\
\hline$e+$ jets $/ \mu$ & 20 & & 3 & 20 & 20 & 140 & - \\
$\mu+$ jets $/ \mu$ & & 15 & 3 & 20 & 20 & 140 & - \\
\hline \hline
\end{tabular}

TABLE II. Efficiency $\times$ branching fraction $(\varepsilon \times \mathcal{B})$ using standard event selection and the expected number of top quark events $(\langle N\rangle)$ in the seven channels, based on the central theoretical $t \bar{t}$ production cross section of Ref. [0], for four top masses. Also given are the expected background, integrated luminosity, and the number of observed events in each channel.

\begin{tabular}{|c|c|c|c|c|c|c|c|c|}
\hline$m_{t}\left(\mathrm{GeV} / c^{2}\right)$ & $e \mu+$ jets & $e e+$ jets & $\mu \mu+$ jets & $e+$ jets & $\mu+$ jets & $e+$ jets $/ \mu$ & $\mu+$ jets $/ \mu$ & ALL \\
\hline$\overline{\varepsilon \times \mathcal{B}(\%)}$ & $0.17 \pm 0.02$ & $0.11 \pm 0.02$ & $0.06 \pm 0.01$ & $0.50 \pm 0.10$ & $0.33 \pm 0.08$ & $0.36 \pm 0.07$ & $0.20 \pm 0.05$ & \\
\hline$\langle N\rangle$ & $1.36 \pm 0.21$ & $1.04 \pm 0.19$ & $0.46 \pm 0.08$ & $4.05 \pm 0.94$ & $2.47 \pm 0.68$ & $2.93 \pm 0.68$ & $1.48 \pm 0.42$ & $13.80 \pm 2.07$ \\
\hline$\varepsilon \times \mathcal{B}(\%)$ & $0.24 \pm 0.02$ & $0.15 \pm 0.02$ & $0.09 \pm 0.02$ & $0.80 \pm 0.10$ & $0.57 \pm 0.13$ & $0.50 \pm 0.08$ & $0.25 \pm 0.06$ & \\
\hline$\langle N\rangle$ & $0.94 \pm 0.13$ & $0.69 \pm 0.12$ & $0.34 \pm 0.07$ & $3.13 \pm 0.54$ & $2.04 \pm 0.53$ & $1.95 \pm 0.39$ & $0.92 \pm 0.24$ & $10.01 \pm 1.41$ \\
\hline$\varepsilon \times \mathcal{B}(\%)$ & $0.28 \pm 0.02$ & $0.17 \pm 0.02$ & $0.10 \pm 0.02$ & $1.20 \pm 0.30$ & $0.76 \pm 0.17$ & $0.56 \pm 0.09$ & $0.35 \pm 0.08$ & \\
\hline$\langle N\rangle$ & $0.57 \pm 0.07$ & $0.40 \pm 0.07$ & $0.19 \pm 0.04$ & $2.42 \pm 0.67$ & $1.41 \pm 0.36$ & $1.14 \pm 0.22$ & $0.64 \pm 0.16$ & $6.77 \pm 1.09$ \\
\hline$\varepsilon \times \mathcal{B}(\%)$ & $0.31 \pm 0.02$ & $0.20 \pm 0.03$ & $0.11 \pm 0.02$ & $1.70 \pm 0.20$ & $0.96 \pm 0.21$ & $0.74 \pm 0.11$ & $0.41 \pm 0.08$ & \\
\hline$\langle N\rangle$ & $0.34 \pm 0.04$ & $0.25 \pm 0.05$ & $0.11 \pm 0.02$ & $1.84 \pm 0.31$ & $0.95 \pm 0.24$ & $0.81 \pm 0.16$ & $0.41 \pm 0.10$ & $4.71 \pm 0.66$ \\
\hline Background & $0.12 \pm 0.03$ & $0.28 \pm 0.14$ & $0.25 \pm 0.04$ & $1.22 \pm 0.42$ & $0.71 \pm 0.28$ & $0.85 \pm 0.14$ & $0.36 \pm 0.08$ & $3.79 \pm 0.55$ \\
\hline $\mathcal{L} d t\left(\mathrm{pb}^{-1}\right)$ & $47.9 \pm 5.7$ & $55.7 \pm 6.7$ & $44.2 \pm 5.3$ & $47.9 \pm 5.7$ & $44.2 \pm 5.3$ & $47.9 \pm 5.7$ & $44.2 \pm 5.3$ & \\
\hline Data & 2 & 0 & 1 & 5 & 3 & 3 & 3 & 17 \\
\hline
\end{tabular}

